\title{
KIAA1377 is associated with lymph node metastasis in esophageal squamous cell carcinoma
}

\author{
SHU-TAO ZHENG ${ }^{1,2^{*}}$, CHEN-CHEN YANG ${ }^{1,2^{*}}$, QING LIU ${ }^{1,2}$, TAO LIU $^{1,2}$, MANG LU $^{1,2}$, \\ FANG DAI $^{1,2}$, XIANG-PENG GAO ${ }^{1,2}$, ILYAR SHEYHIDIN ${ }^{2}$ and XIAO-MEI LU ${ }^{1,2}$ \\ ${ }^{1}$ Clinical Medical Research Institute; ${ }^{2}$ State Key Lab Incubation Base of Xinjiang Major Diseases Research, \\ The First Affiliated Hospital of Xinjiang Medical University, Urumqi, Xinjiang 830011, P.R. China
}

Received July 2, 2015; Accepted September 27, 2016

DOI: $10.3892 / \mathrm{ol} .2016 .5343$

\begin{abstract}
KIAA1377, of which there are few studies regarding cell biology and neurological diseases, has been found to be significantly amplified in esophageal squamous cell carcinoma (ESCC) with lymph node metastasis compared with ESCC without lymph node metastasis. This suggests that KIAA1377 may play a role in the lymph node metastasis of ESCC. There has, to the best of our knowledge, been no study performed to investigate the role of KIAA1377 in ESCC. In the present study, the expression of KIAA1377 was detected by immunohistochemistry, and its expression was statistically analyzed with clinicopathological parameters, using commercially obtained tissue arrays consisting of 86 cases of ESCC and 79 paired controls. KIAA1377 was knocked down ex vivo using transient transfection with specific small hairpin RNA (shRNA) vectors into ESCC TE-1 and EC9706 cell lines whose endogenous KIAA1377 level was highest. The variation of proliferation, migration and invasion were evaluated using methyl thiazolyl tetrazolium, wound healing and Transwell assay, respectively. It was found in vivo that KIAA1377 expression was significantly associated with lymph node metastasis and differentiation, and ex vivo that knockdown of KIAA1377 cannot significantly affect proliferation and mobility in the ESCC cell line TE-1. Overall, this is the first study suggesting that KIAA1377 may play a role in the lymph node micrometastasis of ESCC.
\end{abstract}

\section{Introduction}

Esophageal cancer is one of the most common types of malignant neoplasms and ranks eighth in incidence and

Correspondence to: Dr Xiao-Mei Lu, State Key Lab Incubation Base of Xinjiang Major Diseases Research, The First Affiliated Hospital of Xinjiang Medical University, 137 Liyushan South Road, Urumqi, Xinjiang 830011, P.R. China

E-mail: luxiaomei88@163.com

*Contributed equally

Key words: KIAA1377, lymph node metastasis, ESCC sixth (1) in mortality among cancers worldwide. In China, it ranks fourth (2) in incidence among all cancers and its major pathohistological subtype is esophageal squamous cell carcinoma (ESCC). The majority of esophageal cancer patients hospitalized are often diagnosed with advanced ESCC that metastasizes to local lymph nodes $(3,4)$. Thus, the outcomes and prognosis of patients with ESCC are poor (5), despite the improvements in therapy (6), with the 5-year survival rate being no more than $15 \%$ (7). Therefore, it is necessary to understand the mechanism of lymph node metastasis of ESCC (8).

KIAA serial genes, also termed Cep126, encode large proteins $(9,10)$. The functions of numerous KIAA genes remain unclear, although several studies (11-13) found that certain KIAA genes were involved in certain diseases using DNA microarray or proteomics-based approaches. KIAA1377 was first reported to be localized to the midbody in the cytoplasm, which may govern the process of cytokinesis through forming protein complexes with other midbody proteins (14). Subsequently, Tipton et al (15) found that KIAA1377 is a novel centrosomal protein that also associates with microtubules and the midbody. Another study reported KIAA1377 as a susceptible gene for monomelic amyotrophy (16). There have been no relevant studies on possible roles mediated by KIAA1377 in the neoplasm.

In our previous study (Zheng et al, unpublished data), using the comparative genomic hybridization approach it was found that the copy numbers of the KIAA1377 gene were significantly amplified in ESCC tissues that metastasized to local lymph nodes as compared to ESCC without metastasis (Zheng et al, unpublished data), suggesting that KIAA1377 may play a role in the lymph node metastasis of ESCC. In the present study, the expression of KIAA1377 in ESCC clinical samples and cell lines and its potential roles in metastasis were examined.

\section{Materials and methods}

Tissue microarray. The present study was approved by the Medical Ethics Committee of the First Affiliated Hospital of Xinjiang Medical University (Urumqi, China). The ESCC tissue microarray, consisting of 86 cases of ESCC and 78 cases of paired adjacent normal control tissues, was obtained from Shanghai Outdo Biotech Company (Shanghai, China). The 
clinicopathological information accompanying the tissue array, including tumor-node-metastasis grading and staging, were also obtained. Staging and grading were assessed in accordance with the World Health Organization 2014 version classification and grading system (17). Prognosis and demographics were all available.

Cell culture and transfection. The human ESCC ECa109, EC9706, TE-1, KYSE-30, KYSE-150, KYSE-450 and KYSE-510 cell lines were grown in RPMI-1640 medium supplemented with $10 \%$ fetal bovine serum (FBS) and penicillin/streptomycin in a $5 \% \mathrm{CO}_{2}$ humidified incubator at $37^{\circ} \mathrm{C}$. For knockdown experiments, KIAA1377 short hairpin RNA (shRNA) vector and shRNA scramble control were purchased from Santa Cruz Biotechnology, Inc. (cat. no. sc-96891-SHH, Dallas, TX, USA). ESCC cells were grown to $80-90 \%$ confluence in 6-well plates and were transfected with $4.0 \mu \mathrm{g}$ of shRNA-KIAA1377 vector or control vector together with $10 \mu$ l Lipofectamine 2000 according to the manufacturer's protocol (Invitrogen; Thermo Fisher Scientific, Inc., Waltham, MA, USA). Cells were grown for two days, and media was changed daily.

Immunohistochemistry (IHC). Immunohistochemical stains were performed using heat-induced epitope retrieval (18) and avidin-biotin complex (19) method. The rabbit anti-human KIAA1377 polyclonal antibody (clone, N-16; catalogue no. sc-168347; Santa Cruz Biotechnology, Inc.) was diluted at 1:200. The sections were evaluated by light microscopy, and cellular localization of the protein and immunostaining level in each section was assessed by 2 pathologists. The staining patterns were scored as follows: negative, defined as $<15 \%$; weak ( $\geq 15 \%$ but $<30 \%$ of cells with positive staining), defined as + ; moderate $(\geq 30 \%$ but $<60 \%$ ) of cells with positive staining), defined as ++ ; and strong positive ( $\geq 60 \%$ of cells with positive staining), defined as +++ , according to the immunostaining intensity area.

Reverse transcription-quantitative polymerase chain reaction (RT-qPCR). Total RNA was extracted from frozen tissue with TRIzol reagent (Invitrogen; Thermo Fisher Scientific, Inc.) and reverse transcribed to cDNA using the Prime Script RT-qPCR kit (Takara Biotechnology Co., Ltd., Dalian, China). The RT-qPCR assay was performed with the IQ5 system (Bio-Rad Laboratories, Inc., Hercules, CA, USA) using SYBR Premix Ex Taq (Takara Biotechnology Co., Ltd.), according to the manufacturer's protocol. All reactions were performed in triplicate. Subsequent to normalization with $\beta$-actin controls, relative gene expression was determined using a comparative standard curve. PCR was performed with the following primer sets: KIAA1377 forward, 5'-TCC TAACACAGCCCTAAATGC-3' and reverse, 5'-TCCAGA TTGTAAAGCGTCCAG-3'; and $\beta$-actin forward, 5'-TGG CACCCAGCACAATGAA-3' and reverse, 5'-CTAAGT CATAGTCCGCCTAGAAGCA-3'. The reaction mixtures for KIAA1377 and $\beta$-actin were incubated at the following thermal cycling conditions: $95^{\circ} \mathrm{C}$ for $3 \mathrm{~min} ; 40$ cycles at $95^{\circ} \mathrm{C}$ for $5 \mathrm{sec}$; and $58^{\circ} \mathrm{C}$ for $30 \mathrm{sec}$. the final relative expression of KIAA1377 was calculated after normalization to $\beta$-actin, as internal loading control, using standard curve RT-qPCR approach (20).
Western blot analysis. In total, $72 \mathrm{~h}$ after transfection, TE-1 and EC-9706 cells were harvested in radioimmunoprecipitation assay lysis buffer (BioTeke Corporation, Beijing, China) and $80 \mu \mathrm{g}$ of cellular protein was subjected to $8 \%$ sodium dodecyl sulfate-polyacrylamide gel electrophoresis separation. Proteins were transferred to polyvinylidene fluoride microporous membrane (EMD Millipore, Billerica, MA, USA) and blots were probed with go at polyclonal antibody against human KIAA1377 (catalogue no. sc-168347; dilution at 1:800) and mouse monoclonal antibody against human glyceraldehyde 3-phosphate dehydrogenase (GAPDH; catalogue no. sc-25778; dilution at 1:500) were obtained from Santa Cruz Biotechnology Inc. GAPDH was chosen as an internal control and the blots were visualized with Western Breeze kit (catalogue no., WB7105; Invitrogen; Thermo Fisher Scientific, Inc.), according to the manufacturer's protocol.

Methyl thiazolyl tetrazolium (MTT) assay. MTT spectrophotometric dye assay (Sigma-Aldrich; Merck Millipore, Darmstadt, Germany) was used to observe and compare cell proliferation ability. TE-1 and EC-9706 cells were plated in 96-well plates at a density of $5 \times 10^{3}$ cells per well. Subsequent to transfection experiments, cell proliferation was assessed. Cells were incubated for $4 \mathrm{~h}$ in $20 \mu \mathrm{MTT}$ at $37^{\circ} \mathrm{C}$. The color was developed by incubating the cells in $150 \mu \mathrm{l}$ dimethyl sulfoxide; the absorbance was detected at $490 \mathrm{~nm}$ wavelength. The data were obtained from 3 independent experiments.

Cell migration and invasion assays in vitro. Cell migration ability was calculated by the wound-healing assay. TE-1 and EC-9706 cells were plated in 6-well plates at a concentration of $4 \times 10^{5}$ cells/well and allowed to form a confluent monolayer for $24 \mathrm{~h}$. Subsequent to the transfection experiment, the monolayer was scratched with a sterile pipette tip $(10 \mu \mathrm{l})$, washed with serum free medium to remove floated and detached cells and images were captured (at 0 and $48 \mathrm{~h}$ ) by an inversion fluorescence microscope (Olympus Corporation, Tokyo, Japan). Cell culture inserts (24-well, pore size $8 \mu \mathrm{m}$; BD Biosciences, Franklin Lakes, NJ, USA) were seeded with $5 \times 10^{3}$ cells in $100 \mu \mathrm{l}$ of medium with $0.1 \%$ FBS. Inserts pre-coated with Matrigel (40 $\mu \mathrm{l}, 1 \mathrm{mg} / \mathrm{ml}$; BD Biosciences) were used for invasion assays. Medium with $10 \%$ FBS $(400 \mu \mathrm{l})$ was added to the lower chamber and served as a chemotactic agent. Noninvasive cells were wiped from the upper side of the membrane and cells on the lower side were fixed in cold methanol $\left(-20^{\circ} \mathrm{C}\right)$ and air-dried. Cell were stained with $0.1 \%$ crystal violet (dissolved in methanol) and counted using the inverted microscope. Each individual experiment had triplicate inserts, and 4 microscopic fields (magnification, x100) were counted per insert.

Statistical analysis. Data were expressed as the mean \pm standard deviation and were analyzed by Student's t-test, one-way analysis of variance and $\chi^{2}$ test, as appropriate, using SPSS for Windows version 16.0 (SPSS, Inc., Chicago, IL, USA). Kaplan-Meier survival curves were plotted and log rank test was performed. The significance of various variables for survival was analyzed by Cox proportional hazards model in a multivariate analysis. $\mathrm{P}<0.05$ was considered to indicate a statistically significant difference. 
Table I. Association between KIAA1377 expression and clinicopathological characteristics of ESCC.

\begin{tabular}{|c|c|c|c|c|c|}
\hline \multirow[b]{2}{*}{ Characteristic } & \multirow[b]{2}{*}{ Total, n } & \multicolumn{2}{|c|}{ KIAA1377 expression } & \multirow[b]{2}{*}{$\chi^{2}$} & \multirow[b]{2}{*}{ P-value } \\
\hline & & High $(++,+++)$ & Low $(-,+)$ & & \\
\hline Tissue type & & & & 41.539 & 0.001 \\
\hline Adjacent normal tissues & 78 & 1 & 77 & & \\
\hline ESCC & 86 & 38 & 48 & & \\
\hline Gender & & & & 1.286 & 0.257 \\
\hline Male & 64 & 26 & 38 & & \\
\hline Female & 22 & 12 & 10 & & \\
\hline Age & & & & 1.008 & 0.315 \\
\hline$>60$ years & 57 & 23 & 34 & & \\
\hline$\leq 60$ years & 29 & 15 & 14 & & \\
\hline Clinical stage & & & & 4.807 & 0.090 \\
\hline I & 6 & 2 & 4 & & \\
\hline II & 43 & 24 & 19 & & \\
\hline III & 32 & 10 & 22 & & \\
\hline $\mathrm{N}$ classification & & & & 4.965 & 0.043 \\
\hline $\mathrm{N}_{0}$ & 50 & 27 & 23 & & \\
\hline $\mathrm{N}_{1-3}$ & 34 & 10 & 24 & & \\
\hline T classification & & & & 1.235 & 0.379 \\
\hline $\mathrm{T}_{1}$ & 5 & 1 & 4 & & \\
\hline $\mathrm{T}_{2-4}$ & 79 & 35 & 42 & & \\
\hline Differentiation & & & & 6.999 & 0.030 \\
\hline Well & 8 & 4 & 4 & & \\
\hline Moderately & 60 & 31 & 29 & & \\
\hline Poorly & 18 & 3 & 15 & & \\
\hline Tumor volume & & & & 1.828 & 0.401 \\
\hline$<10 \mathrm{~cm}^{3}$ & 22 & 6 & 16 & & \\
\hline $10-20 \mathrm{~cm}^{3}$ & 27 & 11 & 16 & & \\
\hline$>20 \mathrm{~cm}^{3}$ & 21 & 5 & 16 & & \\
\hline Gross classification & & & & 1.855 & 0.603 \\
\hline Ulcerative type & 45 & 20 & 25 & & \\
\hline Fungating type & 6 & 2 & 4 & & \\
\hline Medullary type & 24 & 11 & 13 & & \\
\hline Protrude type & 2 & 0 & 2 & & \\
\hline
\end{tabular}

ESCC, esophageal squamous cell carcinoma; N, node; T, tumor.

\section{Results}

KIAA1377 expression was significantly upregulated in ESCC tissues and was associated with lymph node metastasis and differentiation. To understand the clinicopathological significance of KIAA1377 expression, a total of 78 distal normal tissues and 86 ESCC tissues were analyzed for KIAA1377 expression using IHC. Expression of KIAA1377 was heterogeneous in ESCC tissues, ranging from weak to moderate to strong positive staining. All paired normal control tissues had positive expression of KIAA1377 compared with ESCC tissues. It can be observed that KIAA1377 positive staining localized at the cytoplasm region of tumor and normal mucosal cells (Fig. 1). Representative results of KIAA1377 expression are shown in Fig. 1. Clinicopathological analyses of KIAA1377 expression are shown in Table I. The KIAA1377 expression level is significantly different in ESCC and normal tissues $(\mathrm{P}=0.001)$. There was a significant difference of KIAA1377 expression levels when the tumor samples were classified based on differentiation $(\mathrm{P}=0.030)$ and lymph node metastases $(\mathrm{P}=0.043)$. No significant association was found between KIAA1377 expression and other parameters (Table I).

To confirm whether or not there was an association between KIAA1377 expression and prognosis, the Kaplan-Meier survival curve for different expression statuses of KIAA1377 was used. Based on 86 patients with ESCC, no significant difference in survival was found comparing patients with weak, moderate or strong KIAA1377 staining in tumors 


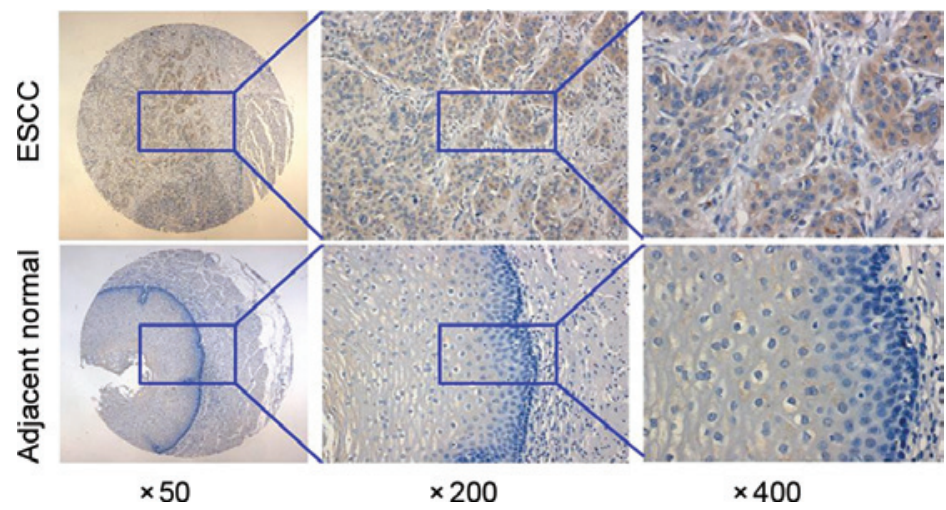

Figure 1. KIAA1377 was significantly upregulated in ESCC tissues compared with the normal control. Immunohistochemical analysis of expression of KIAA1377 in ESCC tissues is shown. ESCC, esophageal squamous cell carcinoma.
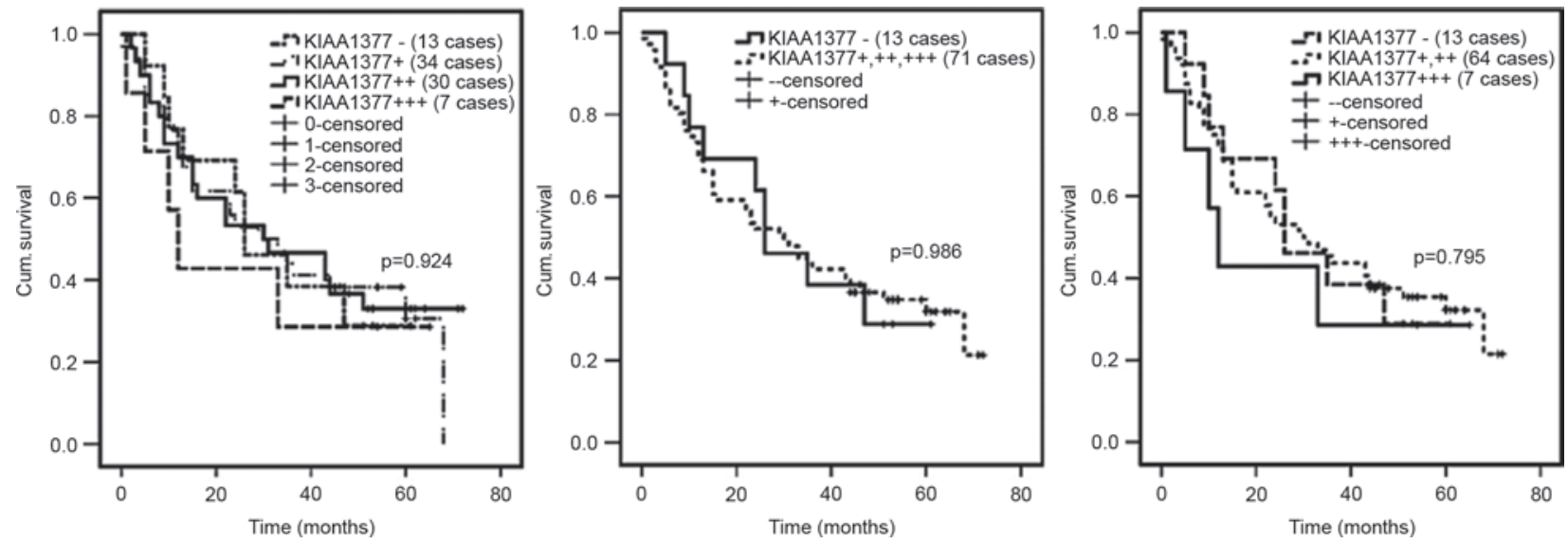

Figure 2. KIAA1377 expression was not significantly associated with prognosis in ESCC. Immunostaining of KIAA1377 and overall survival in ESCC was analyzed by Kaplan-Meier survival curves. There was no significant difference between KIAA1377 expression and prognosis $(\mathrm{P}>0.05)$ subsequent to statistical analysis. ESCC, esophageal squamous cell carcinoma; -, negative; +, weak; ++, moderate; +++, strong; Cum., cumulative.

A

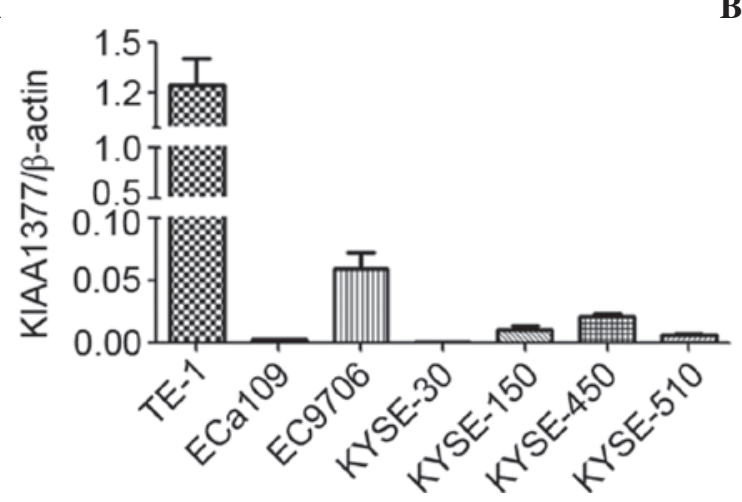

B

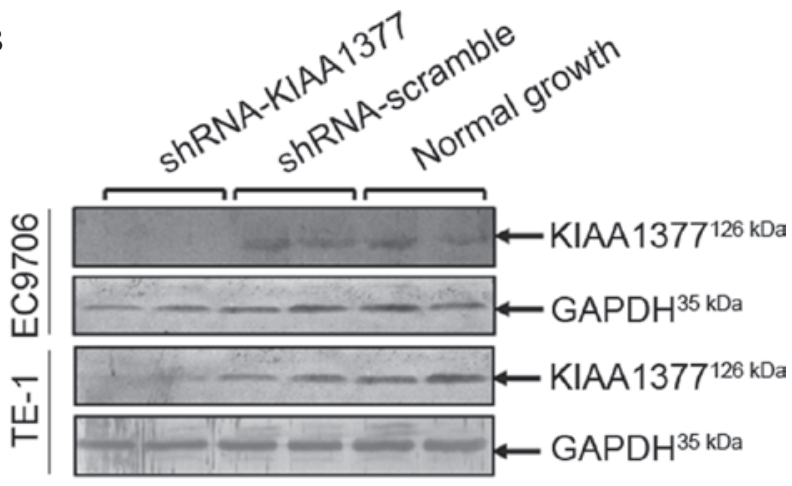

Figure 3. Endogenous basal expression of KIAA1377 in a panel of 7 different ESCC cell lines and evaluation of knockdown efficiency in the TE-1 cell line. (A) Endogenous basal expression of KIAA1377 was detected by reverse transcription-polymerase chain reaction, and $\beta$-actin was used as the internal control. (B) Knockdown efficiency of shRNA-KIAA1377 and shRNA-scramble vector was determined using western blotting. The shRNA-KIAA1377 and shRNA-scramble vector were transfected into ESCC cell line TE-1. In total, $80 \mu \mathrm{g}$ of protein was loaded per lane, which was separated by $8 \%$ sodium dodecyl sulfate-polyacrylamide gel electrophoresis followed by visualization with Western Breeze kit. ESCC, esophageal squamous cell carcinoma; shRNA, small hairpin RNA; GAPDH, glyceraldehyde 3-phosphate dehydrogenase.

( $\mathrm{P}=0.924)$ (Fig. 2). Similarly, multivariate analysis showed there were no significant differences in survival without lymph node metastasis $\left(\mathrm{N}_{0}\right)$ and with lymph node metastasis $\left(\mathrm{N}_{1-3}\right)$ or between stage I and II KIAA1377 immunostaining in tumors (data not shown).
Knockdown of KIAA1377 cannot significantly affect the proliferation, migration and invasion of ESCC cells. To determine the basal expression of KIAA1377 in a panel of ESCC cell lines, RT-qPCR was performed on a panel of 7 different types of ESCC cell lines. It was found that in TE-1 


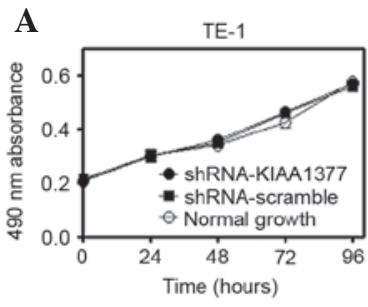

D

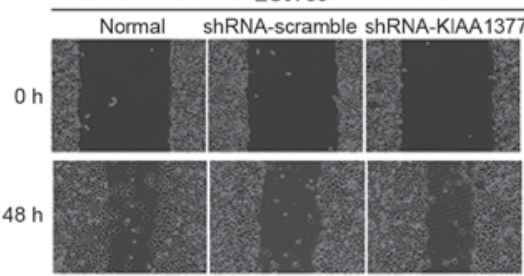

B
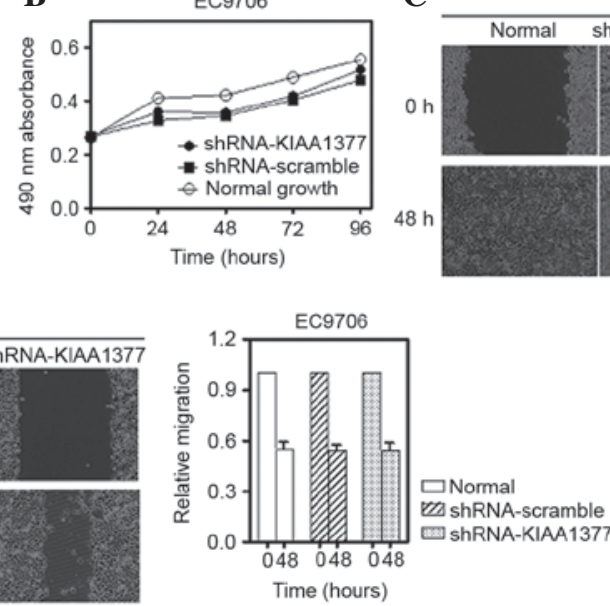

C

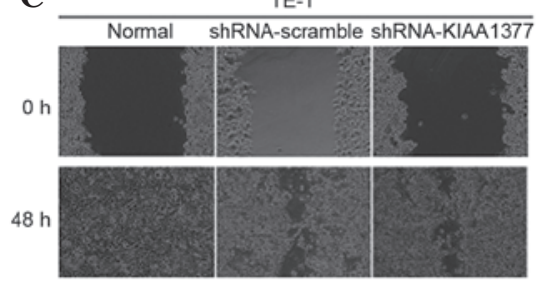

E

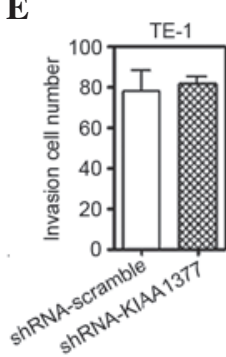

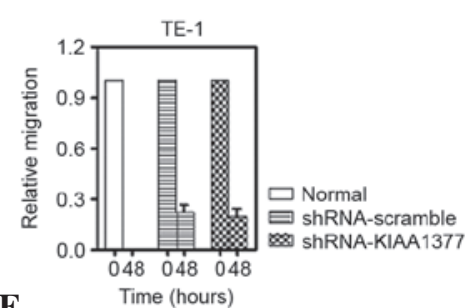

F

EC9706

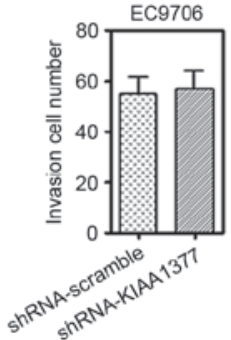

Figure 4. KIAA1377 does not affect the proliferation, migration and invasion ability of cells subsequent to transient knockdown. (A) Methyl thiazolyl tetrazolium assay for the ESCC TE-1 and EC9706 cell lines, (B) subsequent to knockdown of KIAA1377 using transient transfection with a specific shRNA vector for 0, 24, 48, 72 and 96 h. (C) Qualitative wound-healing assay (left) and quantitative assay (right) for TE-1. (D) Similarly, qualitative wound-healing assay (left) and quantitative assay (right) for EC9706 subsequent to transient transfection for 48 h. (E) Quantitative Transwell assay for TE-1 and EC9706, (F) subsequent to knockdown for $48 \mathrm{~h}$ ( $\mathrm{P}>0.05$ compared with control group). Images of migratory cells from the scratched boundary were observed and acquired by light microscopy (magnification, 10x10). Similar results were obtained in 3 independent experiments, and representative images are shown. ESCC, esophageal squamous cell carcinoma.

and EC9706, the 2 different ESCC cell lines, endogenous mRNA level of KIAA1377 were higher than that in other cell lines (Fig. 3A). To understand the role of KIAA1377 in the metastasis of ESCC, specific short hairpin RNA (shRNA) interference vectors were transfected into TE-1 and EC9706 cells. The knockdown efficiency was evaluated using western blot analysis. It was shown that the shRNA vectors against KIAA1377 may effectively knockdown the KIAA1377 protein level (Fig. 3B). Based on which, the proliferation, migration and invasion were assessed by MTT, wound healing and Transwell assays, respectively. It was observed that knockdown of KIAA1377 hardly affected the proliferation (Fig. 4A and B), migration (Fig. 4C and D) and invasion (Fig. 4E and F) abilities subsequent to statistical analysis.

\section{Discussion}

In the present study, it was found that KIAA1377 expression was significantly associated with lymph node metastasis and differentiation in ESCC clinical samples, whereas to the best of our knowledge was not required in the proliferation and mobility ex vivo in ESCC cell lines.

The molecular variations underlying the lymph node metastasis of ESCC remain largely unclear. In our previous study (Zheng et al, unpublished data), differentially expressed genes were screened between ESCC tissues that metastasized to lymph nodes and ESCC tissues without metastasis using comparative genomic hybridization approach. It was found that the copy number of several genes, including KIAA1377, was significantly amplified in ESCC tissues with lymph node metastasis post-surgery as compared with control. Due to the rarity of data of KIAA1377 in cancers and its highest frequency of copy number variation, KIAA1377 was targeted among the differential genes screened out and hypothesized that amplification of KIAA1377 may facilitate ESCC cells metastasis into lymph nodes. Based on the hypothesis, it has firstly confirmed the expression of KIAA1377 using IHC with ESCC tissue array. It was identified that KIAA1377 expression was significantly associated with lymph node metastasis and cell differentiation degree, which was consistent with our previous finding on copy number variations. However, no significant association was observed between KIAA1377 expression and prognosis, as well as other clinicopathological parameters.

To additionally verify the supposed role of KIAA1377 in facilitating the lymph node metastasis in ESCC, KIAA1377 was knocked down in TE-1 cells, which demonstrated the higher basal expression level among the 7 different ESCC cell lines available. It was shown that KIAA1377 has little effect on the proliferation, migration and invasion subsequent to transient knockdown, suggesting that KIAA1377 maybe dispensable in the proliferation and mobility of ESCC cell lines ex vivo.

With regard to the role of KIAA1377 in lymph node metastasis, there appears to be somewhat controversial and inconsistent findings in vivo and ex vivo. However, in consideration of studies (14-16) available regarding the way KIAA1377 works in physiological or pathophysiological setting, KIAA1377 may co-ordinate with other unknown proteins $(14,21)$ located on the midbody or in the cytoplasm through protein-protein interactions $(15,21,22)$. Thus it would make sense that only knockdown of KIAA1377 may cause few phenotypic defects as other compensation mechanisms maybe provided by other interacting proteins $(23,24)$. Furthermore, Bonavita et al (25) found that depletion of Cep-126, also known as KIAA1377, was required for the formation of primary cilium in hTERT-RPE-1 and IMCD3 cells. Based on this study, it is hypothesized that KIAA1377 may be associated with micro-metastasis in ESCC cells. Therefore, it may be due to the detection limit in the 
experimental setting that no significant phenotypic variation was observed ex vivo subsequent to knockdown of KIAA1377 in ESCC cells.

Despite the limited cases studied and lack of mechanistic information provided by in vitro KIAA1377 experiments, to the best of our knowledge, this is the first study that shows KIAA1377 is significantly associated with lymph node metastasis and differentiation in ESCC, but is dispensable in the motility of ESCC cell lines ex vivo. Overall, KIAA1377 may play a role in the lymph node micrometastasis of ESCC.

\section{Acknowledgements}

The present study was supported by National Science Foundation of China (grant no. 81360357). The authors thank Dr Antonino Colanzi [Institute of Protein Biochemistry (CNR), Naples, Italy] and Associate Professor Songtao Liu (Department of Biological Sciences, University of Toledo, $\mathrm{OH}$, USA) for constructive proofreading and comments.

\section{References}

1. Torre LA, Bray F, Siegel RL, Ferlay J, Lortet-Tieulent J and Jemal A: Global cancer statistics, 2012. CA Cancer J Clin 65: 87-108, 2015.

2. Chen W, Zheng R, Baade PD, Zhang S, Zeng H, Bray F, Jemal A, Yu XQ and He J: Cancer statistics in China, 2015. CA Cancer J Clin 66: 115-132, 2016.

3. Pech O: Nodes or no nodes? The lymph node metastasis risk of T1 esophageal cancer revisited. J Natl Cancer Inst 106: dju174, 2014.

4. Vidovic V, Nikolic I, Vukojević J, Samardzija G, Kukic B, Bogdanović B and Petrović N: Unusual metastasis of esophageal cancer. Vojnosanit Pregl 71: 975-977, 2014.

5. Lin D and Leichman L: The current status of neoadjuvant therapy for esophageal cancer. Semin Thorac Cardiovasc Surg 26: 102-109, 2014.

6. Gaur P, Kim MP and Dunkin BJ: Esophageal cancer: Recent advances in screening, targeted therapy, and management. J Carcinog 13: 11, 2014.

7. Ford HE: Gefitinib for oesophageal cancer: A cog in need of a wheel? Lancet Oncol 15: 790-791, 2014.

8. He Z, Wu S, Li Q, Lin Q and Xu J: Use of the metastatic lymph node ratio to evaluate the prognosis of esophageal cancer patients with node metastasis following radical esophagectomy. PloS One 8: e73446, 2013.

9. Koga H, Shimada K, Hara Y, Nagano M, Kohga H, Yokoyama R, Kimura Y, Yuasa S, Magae J, Inamoto S, et al: A comprehensive approach for establishment of the platform to analyze functions of KIAA proteins: Generation and evaluation of anti-mKIAA antibodies. Proteomics 4: 1412-1416, 2004.

10. Nakajima D, Okazaki N, Yamakawa H, Kikuno R, Ohara O and Nagase T: Construction of expression-ready cDNA clones for KIAA genes: Manual curation of 330 KIAA cDNA clones. DNA Res 9: 99-106, 2002.
11. Mete O, Lopes MB and Asa SL: Spindle cell oncocytomas and granular cell tumors of the pituitary are variants of pituicytoma. Am J Surg Pathol 37: 1694-1699, 2013.

12. Zhang KQ, Salzman SA, Reding DJ, Suarez BK, Catalona WJ and Burmester JK: Genetics of prostate cancer. Clin Med Res 1: 21-28, 2003.

13. Sugita Y, Nakamura Y, Yamamoto M, Ogasawara S, Ohshima K and Shigemori M: Expression of KIAA 0864 protein in neuroepithelial tumors: An analysis based on the presence of monoclonal antibody HFB-16. J Neurooncol 89: 151-158, 2008.

14. Chen TC, Lee SA, Hong TM, Shih JY, Lai JM, Chiou HY, Yang SC, Chan CH, Kao CY, Yang PC and Huang CY: From midbody protein-protein interaction network construction to novel regulators in cytokinesis. J Proteome Res 8: 4943-4953, 2009.

15. Tipton AR, Wang K, Oladimeji P, Sufi S, Gu Z and Liu ST: Identification of novel mitosis regulators through data mining with human centromere/kinetochore proteins as group queries. BMC Cell Biol 13: 15, 2012.

16. Lim YM, Koh I, Park YM, Kim JJ, Kim DS, Kim HJ, Baik KH, Choi HY, Yang GS, Also-Rallo E, et al: Exome sequencing identifies KIAA1377 and C5orf42 as susceptibility genes for monomelic amyotrophy. Neuromuscul Disord 22: 394-400, 2012.

17. McGuire S: World Cancer Report 2014. Geneva, Switzerland: World Health Organization, International Agency for Research on Cancer, WHO Press, 2015. Adv Nutr 7: 418-419, 2016.

18. Shi SR, Shi Y and Taylor CR: Antigen retrieval immunohistochemistry: Review and future prospects in research and diagnosis over two decades. J Histochem Cytochem 59: 13-32, 2011.

19. Cardiff RD, Miller CH and Munn RJ: Manual immunohistochemistry staining of mouse tissues using the avidin-biotin complex (ABC) technique. Cold Spring Harb Protoc 2014: 659-662, 2014

20. Percopo CM, Dyer KD, Karpe KA, Domachowske JB and Rosenberg HF: Eosinophils and respiratory virus infection: A dual-standard curve qRT-PCR-based method for determining virus recovery from mouse lung tissue. Methods Mol Biol 1178: 257-266, 2014.

21. Murakami M, Shimada K, Kawai M and Koga H: InCeP: Intracellular pathway based on mKIAA protein-protein interactions. DNA Res 12: 379-387, 2005.

22. Hermjakob H, Montecchi-Palazzi L, Bader G, Wojcik J, Salwinski L, Ceol A, Moore S, Orchard S, Sarkans U, von Mering C, et al: The HUPO PSI's molecular interaction format-a community standard for the representation of protein interaction data. Nat Biotechnol 22: 177-183, 2004.

23. Hall EA, Keighren M, Ford MJ, Davey T, Jarman AP, Smith LB, Jackson IJ and Mill P: Acute versus chronic loss of mammalian Azi1/Cep131 results in distinct ciliary phenotypes. PLoS Genet 9: e1003928, 2013.

24. Stelzl U, Worm U, Lalowski M, Haenig C, Brembeck FH, Goehler H, Stroedicke M, Zenkner M, Schoenherr A, Koeppen S, et al: A human protein-protein interaction network: A resource for annotating the proteome. Cell 122: 957-968, 2005.

25. Bonavita R, Walas D, Brown AK, Luini A, Stephens DJ and Colanzi A: Cep126 is required for pericentriolar satellite localisation to the centrosome and for primary cilium formation. Biol Cell 106: 254-267, 2014. 